\title{
Efficient Melt Stirring Using Pulse Sequences of a Rotating Magnetic Field: Part I. Flow Field in a Liquid Metal Column
}

\author{
S. ECKERT, P. A. NIKRITYUK, D. RÄBIGER, K. ECKERT, and G. GERBETH
}

DOI: $10.1007 / \mathrm{s} 11663-008-9147-5$

(C) The Minerals, Metals \& Materials Society and ASM International 2008

Erratum to: METALLURGICAL AND MATERIALS

TRANSACTIONS B, Vol. 38B, No. 6,

December 2007

DOI: $10.1007 / \mathrm{s} 11663-007-9096-4$

THE color figures in this article were inadvertently printed in black and white in the printed issue.

S. ECKERT, Senior Research Scientist, D. RÄBIGER, Graduate Student, and G. GERBETH, Group Leader, are with the MHD Department, Forschungszentrum Dresden-Rossendorf (FZD), D-01314, Dresden, Germany. Contact e-mail: s.eckert@fzd.de P.A. NIKRITYUK, Postdoctoral Fellow, and K. ECKERT, Group Leader, are with the Institute for Aerospace Engineering, Dresden University of Technology, D-01062, Dresden, Germany.

The online version of the original article can be found under doi: 10.1007/s11663-007-9096-4.

Article published online April 30, 2008. 


\title{
Efficient Melt Stirring Using Pulse Sequences of a Rotating Magnetic Field: Part I. Flow Field in a Liquid Metal Column
}

\author{
S. ECKERT, P.A. NIKRITYUK, D. RÄBIGER, K. ECKERT, and G. GERBETH
}

The use of a pulsed, rotating magnetic field (RMF) is presented as an auspicious method for obtaining an intensive stirring and mixing in a pool of liquid metal; the RMF pulses within a sequence have been applied with a constant or alternating direction. The resulting flow structure in a cylindrical liquid metal column has been explored by numerical simulations and by model experiments, using the ternary alloy GaInSn. Ultrasonic Doppler velocimetry (UDV) has been used to determine profiles of the vertical velocity. Both the numerical results and the velocity measurements demonstrate the capability of the proposed stirring regimes for overcoming the limited mixing character of conventional rotary stirring. The application of a time-modulated RMF offers considerable potential for providing an optimal flow pattern in a solidifying melt, for reasons of a well-aimed modification of casting properties.

DOI: $10.1007 / \mathrm{s} 11663-007-9096-4$

(C) The Minerals, Metals \& Materials Society and ASM International 2007

\section{INTRODUCTION}

Alternating current (AC) magnetic fields are commonly used in industrial practice for melt stirring. ${ }^{[1-6]}$ The requirements arising from the particular metallurgical or casting operation are manifold. For example, the electromagnetic stirring should provide an efficient mixing of the melt or counterbalance buoyancy-driven flows. In principle, different magnetic fields (rotating, traveling, pulsating, and combinations of these three) are available, but each field type gives rise to a more or less symptomatic flow pattern. For the optimization of melt-stirring operations, a suitable magnetic field design has to be chosen. Furthermore, the magnetic-field parameters (amplitude, frequency, and spatial and temporal structure) have to be adjusted according to the requirements.

In the present article, we consider the standard case of electromagnetic stirring by means of a rotating magnetic field (RMF). The rotary stirring is thought to be employed in steelmaking not only for reasons of ladle mixing or flow control at the mold region in continuous casting, ${ }^{[2-4]}$ but also during the solidification of metal alloys, where it has been proven a powerful method for achieving a purposeful alteration of the microstructure of casting ingots. The RMF-driven convection promotes a transition from a columnar to an equiaxed dendritic growth (CET) and provokes a distinct grain-refining effect. ${ }^{[7,8]}$ However, it was also observed that the RMF stirring of a solidifying metal alloy causes macrosegregations. ${ }^{[9-12]}$

S. ECKERT, Senior Research Scientist, D. RÄBIGER, Graduate Student, and G. GERBETH, Group Leader, are with the MHD Department, Forschungszentrum Dresden-Rossendorf (FZD), D-01314, Dresden, Germany. Contact e-mail: s.eckert@fzd.de P.A. NIKRITYUK, Postdoctoral Fellow, and K. ECKERT, Group Leader, are with the Institute for Aerospace Engineering, Dresden University of Technology, D-01062, Dresden, Germany.

Manuscript submitted May 3, 2007.
In the majority of cases, the RMF stirring is applied as continuous stirring. For a metal alloy in a cylindrical column, the imposition of an RMF leads basically to a swirling motion of the liquid, usually called a primary flow. A secondary recirculating flow in the $r-z$ plane results from the Ekman pumping at the horizontal walls. Although the amplitude of the primary motion is several times higher than the secondary flow, its contribution to an efficient bulk mixing is marginal, because it consists mainly of an almost rigidly rotating core. The convective transport in the vertical and radial directions is mainly achieved by the secondary flow, which consists of a double-vortex structure for the laminar case. Therefore, an amplification of the secondary flow causes an intensification of the mixing rate, which can be realized in the simplest way by increasing the magnetic field. At sufficiently high field intensities, so-called Taylor-Görtler vortices occur, moving along the sidewalls of the cylinder and dissipating inside the Bödewadt layers. Such vortex structures are very efficient with respect to melt mixing. ${ }^{[13]}$ On the other hand, an increase in the magnetic field also accelerates the primary, swirling flow, which results in increasing deflection of the free surface. Instabilities at the meniscus region should be strictly avoided in many applications, because they can lead to surface defects or the entrainment of gas. In addition, specific problems arise if the rotary stirring is applied during directional solidification. For example, for a metal alloy solidified in vertical direction, the double-vortex structure of the secondary flow results in a radial, inward flow along the solidification front. The flow transports the solute toward the axis of the ingot and is, therefore, responsible for the typical segregation pattern in the form of vertical channels filled with eutectic alloy. ${ }^{[9-11]}$ A mitigation of the problem could probably be achieved if the flow in the mushy zone is oriented by turns in different directions. 
Regardless of the differences between the continuous casting process and the unidirectional solidification of metal alloys, these problems related to rotary stirring impede the efficient use of an RMF in both cases. For this reason, a new concept of electromagnetic stirring has to be found. A few techniques are known; they have been proposed to avoid the disadvantages of rotary, electromagnetic stirring mentioned here. These concepts will be introduced in Section II of the present paper. Our approach is to find a method that makes it possible to amplify the secondary, recirculating flow, but to limit the amplitude of the primary, swirling flow at the same time. As a first step toward this goal, we will demonstrate in Part I of this article that a modulated RMF application can enforce a higher mixing rate, both at a lower energy consumption and at a nearby flat surface.

We study the melt flow excited by the application of a time-modulated RMF. In the present article, two specific types of RMF stirring have been considered. First, we used a succession of pulses that always have the same rotational direction, called the RMF pulse sequence of constant direction (RMF-PSCD). Second, we used an RMF pulse sequence of alternating direction (RMF-PSAD); this is related to a periodic inversion of the sense of rotation between two consecutive pulses. An optimization of the electromagnetic stirring with regard to an efficient mixing or a control of the solidification processes requires a profound knowledge of the flow structure; therefore, Part I of this article is exclusively focused on obtaining quantitative information on the flow fields in a circular cylinder generated by a single RMF pulse and a pulse sequence. Here, we consider a model experiment not exclusively related to a particular industrial application. In fact, we want to study how a flow field evolves under isothermal conditions if the liquid metal is agitated by a succession of RMF pulses. The knowledge gained is applied in Part II of this article, which is specifically concerned with the effect of pulsed rotary stirring during the directional solidification of Al$\mathrm{Si}$ alloys. Numerical simulations and experimental investigations have been carried out. Velocity measurements have been realized using the ultrasound Doppler velocimetry (UDV). The experimental setup will be described in Section III, whereas the numerical procedure is briefly introduced in Section IV. Section V is devoted to the specific flow pattern arising from an impulsive change in the magnetic-field strength, known as the spinup or spindown of the flow. Experimental and numerical results with respect to the velocity field during electromagnetic stirring via an RMF pulse sequence of constant or alternating direction will be presented in Sections VI and VII, respectively. The results will be discussed in Section VIII and the conclusions can be found in Section IX.

\section{ADVANCED CONCEPTS OF ROTARY, ELECTROMAGNETIC STIRRING}

Several studies have been devoted to overcoming the handicaps of rotary stirring, with the specific goal of providing a vigorous stirring in the bulk without considerable deformation of the free surface. Vives ${ }^{[6]}$ developed a helical induction stirrer, which generates not only rotational but also vertical forces. For the same reason, Taniguchi et al. ${ }^{[14]}$ proposed a hybrid stirrer that combines the action of a rotating and vertically traveling magnetic field. An electromagnetic stirring system that employs two rotating magnetic fields was considered by Chang et al. ${ }^{[4]}$ Whereas the bulk volume of the mold is agitated by a main electromagnetic stirring (M-EMS) system, a second stirrer, a so-called alternating currentstirring modifier (AC-SM), should control the meniscus region by generating a fluid rotation in the opposite direction. The opposite stirring is supposed to keep the free surface quiescent while a vigorous stirring is maintained in the rest of the pool.

All these approaches require a modification of the stirrer design or the installation of additional induction coils. From an economic point of view, it would be attractive to achieve the same benefit with the alreadyexisting coil system. Spitzer et al. ${ }^{[2]}$ proposed a multifrequency stirring. The authors applied a low-frequency, three-phase current rotating counterclockwise and a high-frequency current rotating in a clockwise direction, whereas the high-frequency part only acts in the exterior domain of the liquid because of the skin effect. It was shown that, for the relevant time-averaged volume force, the superposition principle is valid with respect to the contribution of both frequencies. Kojima et al. ${ }^{[15]}$ adopted a rotary, electromagnetic stirrer to reduce the center segregation in the continuous casting of steel. Because previous plant trials showed that continuous stirring does not inhibit such segregation effects, the authors developed new stirring methods with varying directions and intensities of the magnetic field. One variant, named the differential-phase-current (DPC) stirring, was implemented by a frequency variation of a two-phase current. Another technique is based on a periodic activation of a pulse-shaped RMF, both in intermittent and alternating form. The particular pulses are separated by dormant periods, while the development of the fluid flow is left to its own inertia. The authors performed tests on Wood's metal. They carried out velocity measurements at particular positions in the melt, which revealed an oscillating behavior of the flow. Although a reduction of the center segregation was achieved using these types of stirring, the methods are not considered effective enough to meet the quality requirements for high-grade steels.

An optimized application of an intermittently-applied RMF for melt stirring requires a better understanding of the fluid dynamics in such processes. If a sequence of RMF pulses is imposed on the melt, the resulting flow experiences a succession of spinup and spindown cycles. Analytical and numerical studies concerning the spinup of an RMF-driven flow have been published recently. ${ }^{[16,17]}$ Nikrityuk et al. ${ }^{[18]}$ investigated the transient behavior of the flow arising from the application of a single RMF pulse. Here, the flow is governed by an impulsive spinup from the rest state, followed by a spindown phase resulting from the inertia of the fluid. Under specific conditions determined by the imposition of a pulsed RMF, the secondary, recirculating flow 
shows periodical reversals; a considerable strengthening of the secondary flow with respect to the primary flow can be noticed as well. ${ }^{[18]}$ These findings give reason to anticipate the possibility of overcoming the disadvantages of conventional rotary stirring by means of a pulsed RMF.

The present article focuses on a systematic investigation of flow pattern driven by different types of a timemodulated RMF, whereas the temporal structure of the magnetic field is composed of sequential RMF pulses. A striking method of flow tailoring is in demand for generating favorable flow structures featured by an intensive mixing, while at the same time minimizing both the energy consumption and the interfering perturbations of the free surface. Moreover, the synthesis of a secondary flow with periodic inversions of the flow direction in the vicinity of the solidification front seems to hold promise for avoiding flow-induced macrosegregation during solidification.

\section{EXPERIMENTAL SETUP}

Model experiments are an important tool for understanding the details of the flow structure and the transport properties of flows occurring in real-scale metallurgical facilities and for validating the multitude of numerical codes for the flow simulation. The model experiment presented here should help us to understand how a fluid flow develops under the influence of a sequence of RMF pulses, which has to be considered as a complex fluid dynamical problem. For this reason, we have chosen a low-melting-point liquid metal in a simple geometry, namely, a cylindrical cavity. The flow measurements were carried out using the ternary eutectic alloy Ga68In20Sn12, which is liquid at room temperature. The cylindrical fluid vessel was made from Perspex with a radius $R_{0}$ of $30 \mathrm{~mm}$. The height of the liquid metal column $H_{0}$ was chosen to be $60 \mathrm{~mm}$, ensuring an aspect ratio $2 R_{0} / H_{0}$ of unity. The experiments were carried out in the magnetic induction system KOMMA* at FZD,

*KOMMA is an experimental magnetic induction system built-on by FZD

which is composed of two coil systems that allow for the generation of both rotating and traveling magnetic fields. A schematic view of the experimental setup is depicted in Figure 1. The bore diameter of the magnetic system is $90 \mathrm{~mm}$, wherein the fluid vessel was placed concentrically. In order to preclude flow artifacts arising from symmetry deviations of the experimental setup (vertical alignment, conformity of both the cylinder, and magnetic-field axis), special care was necessary to ensure a precise positioning of the cylinder inside the magnetic system. The RMF was generated by an arrangement of six air-cooled copper coils, which were fed with a threephase electric current. The magnetic system is able to provide field intensities of, at most, $25 \mathrm{mT}$ and a frequency in the domain between 10 and $400 \mathrm{~Hz}$. The homogeneity of the magnetic field was checked using a

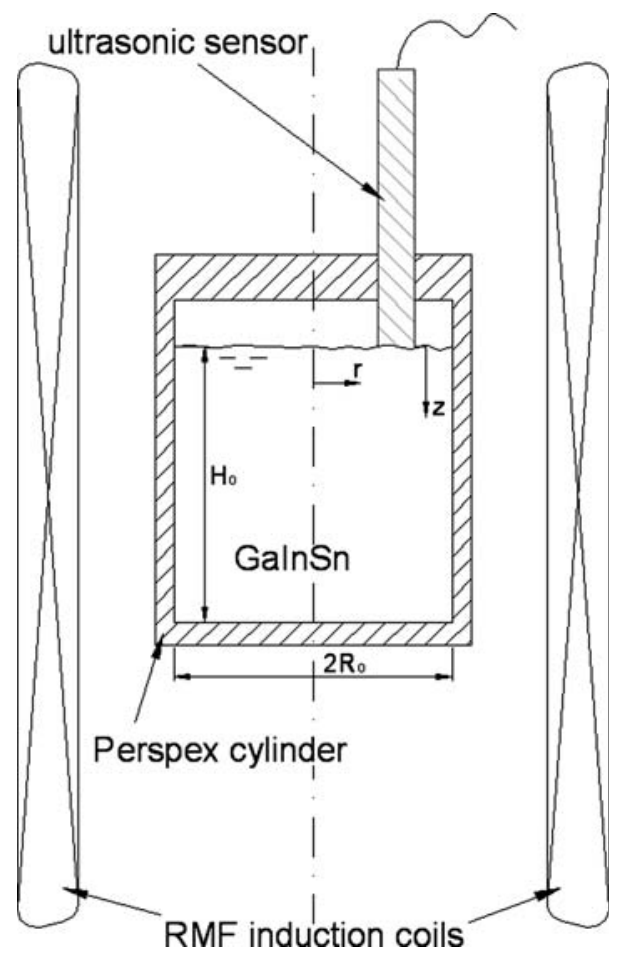

Fig. 1-Schematic view of the experimental setup.

three-axis Gauss meter (Lakeshore model 560, sensor type MMZ2560-UH, Lakeshore). Within a radius of $30 \mathrm{~mm}$, which was selected as the radial dimension of the container, the variance of the magnetic-field strength was found to be smaller than 5 pct. The $50-\mathrm{hZ}$ frequency in the magnetic field has been modulated with sequences of rectangular pulses. The modulation has been carried out by switching the power supply with an external waveform generator. The modulation frequencies (typically below $1 \mathrm{~Hz}$ ) are considerably lower than the magneticfield frequency of $50 \mathrm{~Hz}$. The different variants of the modulation that have been realized in our experiments will be discussed in the subsequent sections of this article.

The UDV, which delivers instantaneous profiles of the local velocity along the ultrasonic beam, is a very attractive technique for obtaining experimental data from flows of opaque liquids. Details concerning the measuring principle can be found in Reference 19. Over the past years, the UDV technique has become an accepted method for flow investigations in various liquid metals - for example, gallium and GaInSn. ${ }^{[20,21]}$ Our measurements have been carried out using the DOP2000 velocimeter (model 2125, Signal Processing SA, Lausanne) equipped with an 8-MHz transducer (TR0805LS, diameter $5 \mathrm{~mm}$, Signal Processing SA, Lausanne). The transducer was aligned vertically and attached to the free surface of the liquid metal. All velocity measurements presented in this article have been carried out at a radial position of $r / R_{0}=0.6$. Using this arrangement, profiles of the vertical velocity $v_{z}$ have been recorded between the front end of the sensor and the bottom of the fluid container. The sensor surface penetrated the melt surface until a depth of about $2 \mathrm{~mm}$. The measuring volume can be considered as a series of consecutive 
discs lined up concentrically along the ultrasonic beam. Because of the divergence of the ultrasonic beam, the lateral size of the measuring volume increases with the distance from the transducer. Hence, the spatial resolution in the lateral direction varies in a range from $5 \mathrm{~mm}$ at the sensor to approximately $7.5 \mathrm{~mm}$ at the bottom of the fluid vessel. In an axial direction, a spatial resolution of about $1.4 \mathrm{~mm}$ was achieved. The velocity data were acquired with a sampling frequency of $8 \mathrm{~Hz}$. The resolution of the velocity data is about $0.15 \mathrm{~mm} / \mathrm{s}$.

\section{NUMERICAL METHOD}

A detailed description of our model and the numerical implementation can be found in Nikrityuk et al. ${ }^{[18]}$ and will be briefly summarized here. The simulations consider an electrically-conducting liquid in a finite cylinder (radius $R_{0}$, height $H_{0}$ ) exposed to an RMF. The rotation of the magnetic field induces a Lorentz force $F_{L}$, which drives the liquid motion. In general, the Lorentz force consists of both a steady axisymmetric part and a timedependent one oscillating with the double frequency. The latter one becomes relevant only for small frequencies $^{[22]}$ and can be neglected for our experimental conditions. Furthermore, the modeling of the Lorentz force presumes both the low-frequency and low-induction approximation. The low-induction approximation implies that both the magnetic Reynolds number $\operatorname{Re}_{m}=\mu_{0} \sigma u R_{0}$ and the magnetic interaction parameter $N=\sigma B_{0}^{2} / \rho \omega$ are much smaller than unity. The lowfrequency approximation requires the skin depth of the magnetic field to be much larger than the radius of the cylinder $R_{0}$ or, in other words, the shielding parameter $S=\mu_{0} \sigma \omega R_{0}^{2}<1$. If both conditions are fulfilled, the Lorentz force has only a nonoscillating part that is unaffected by the fluid motion. In this case, an analytical solution can be used for the Lorentz force that has a predominant component in the azimuthal direction:

$$
\begin{aligned}
F_{L} & =\frac{1}{2} \sigma \cdot \omega \cdot B_{0}^{2} \cdot r \\
& \cdot\left(1-\frac{2}{r^{\prime}} \cdot \sum_{k=1}^{\infty} \frac{J_{1}\left(\lambda_{k} r^{\prime}\right) \cdot \cosh \left(\lambda_{k}\left(z^{\prime}-\frac{1}{2} H^{\prime}\right)\right)}{\left(\lambda_{k}^{2}-1\right) \cdot J_{1}\left(\lambda_{K}\right) \cdot \cosh \left(\frac{1}{2} \lambda_{k} H^{\prime}\right)}\right)
\end{aligned}
$$

where $z^{\prime}=z / R_{0}, r^{\prime}=r / R_{0}, H^{\prime}=H_{0} / R_{0}, J_{1}$ and $J_{2}$ are Bessel functions, and the $\lambda_{k}$ terms are the roots of $J_{1}^{\prime}(x)=0$.

The liquid metal is considered a Newtonian incompressible fluid, which is assumed to be axisymmetric. An open-source code of a Navier-Stokes solver ${ }^{[23]}$ has been adopted to solve the transport equations for mass and momentum:

$$
\begin{gathered}
\nabla \cdot \mathbf{u}=0 \\
\frac{\partial \mathbf{u}}{\partial t}+(\mathbf{u} \nabla) \mathbf{u}=-\frac{1}{\rho} \nabla p+v \Delta \mathbf{u}+\frac{F_{L} \mathbf{e}_{\theta}}{\rho}
\end{gathered}
$$

where the cylindrical polar coordinates $(r, \theta, z)$ are used. Axisymmetry is forced, by cancelling the derivatives with respect to $\theta$. The projection of the momentum conservation equation in the azimuthal direction has the form

$$
\begin{aligned}
\frac{\partial u_{\theta}}{\partial t} & +u_{z} \frac{\partial u_{\theta}}{\partial z}+u_{r} \frac{\partial u_{\theta}}{\partial r}=v \frac{\partial^{2} u_{\theta}}{\partial z^{2}}+\frac{v}{r} \frac{\partial}{\partial r}\left(r \frac{\partial u_{\theta}}{\partial r}\right) \\
& -\frac{u_{r} u_{\theta}}{r}-v \frac{u_{\theta}}{r^{2}}+\frac{F_{L}}{\rho}
\end{aligned}
$$

No-slip conditions on all walls except the top are applied. Due to the open-cavity experimental setup, we use the stress-free condition on the free surface.

The SIMPLE algorithm with an arrangement of collocated variables has been applied, to calculate the pressure and the velocity fields. For the stabilization of the pressure-velocity coupling, the Rhie-Chow scheme has been used. The convection terms are discretized by a deferred-correction scheme. ${ }^{[23]}$ The diffusion terms were approximated, using the second-order central differencing scheme. The time derivatives are discretized by a three-time-level scheme. The system of linear equations is solved by using Stone's strongly-implicit procedure (SIP). Time marching with a fixed time-step was used. The outer iterations for each time-step were stopped when the maximum residual was less than $10^{-10}$ in physical units. Several grid-convergence tests are performed. The final grid has 120 and 240 control volumes in the radial and the axial directions, respectively. The code was validated in Reference 17.

\section{SPINUP AND SPINDOWN OF AN RMF-DRIVEN FLOW}

This section is devoted to the melt flow occurring during a spinup or spindown of an RMF. The knowledge about the respective velocity fields provides a basis for developing techniques for an efficient melt stirring.

Let us consider a stagnant liquid metal in a circular cylinder suddenly exposed to a RMF characterized by the amplitude $B_{0}$ and the frequency $\omega$ during a time period $t_{p}=t_{1}-t_{0} \quad$ (Figure 2(a)). Figure 2(b) shows what occurs when the sense of rotation of the field vector is inverted after the RMF pulse. This situation will be discussed at the end of this section. At the time $t_{0}$, the Lorentz force $F_{L}$ starts to accelerate the liquid. This process is called spinup. The Lorentz force is not uniform along the axial direction, and shows a maximum at the horizontal midplane of the cylinder. Therefore, the fluid rotates faster here compared to the regions at the top and the bottom, where the velocity declines to zero at the wall across the developing Bödewadt boundary layers. The consequence is an imbalance of the centrifugal forces driving a radial outward flow at the horizontal midplane. For reasons of continuity, a secondary circulation is formed in the meridional plane, which appears as two toroidal vortices. This first period of the spinup, in which viscous effects play only a negligible role, is also called the initial adjustment phase. ${ }^{[17]}$ The nonlinear interaction between the primary and secondary flow becomes important in the subsequent inertial phase. The development of the 


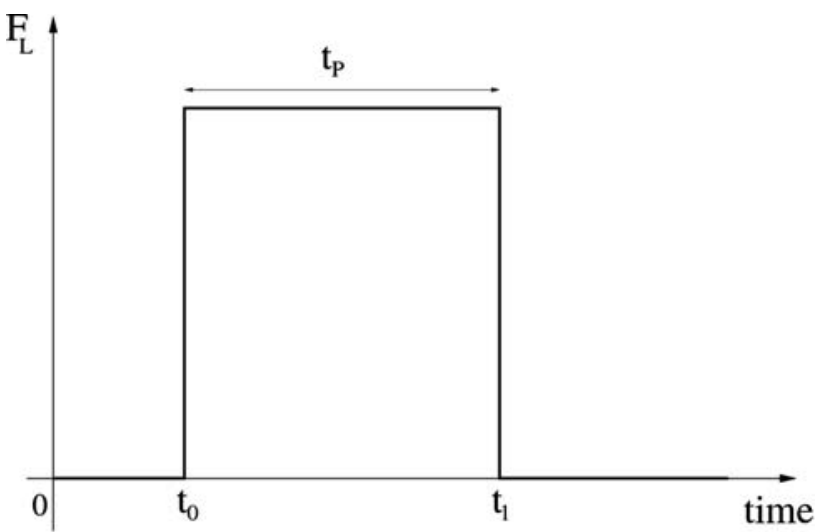

(a)

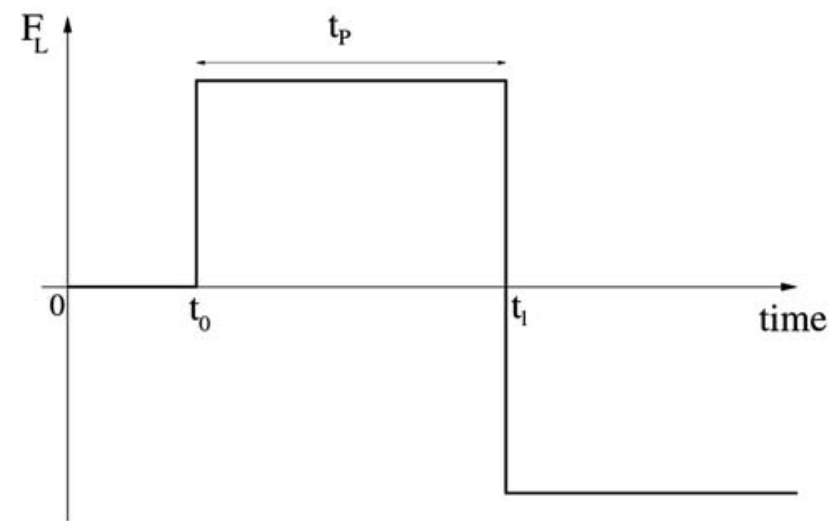

(b)

Fig. 2-Scheme of the applied RMF pulse: $(a)$ single pulse or $(b)$ pulse with subsequent reversal of the direction of the RMF.

secondary flow proceeds via inertial oscillations with the two-toroidal vortex pattern of the previous initial adjustment phase. As a result, the multilayered structure of the Bödewadt layers at the horizontal endwalls is established. For the supercritical values of the magnetic induction, Taylor-Görtler vortices appear near the sidewall layers and move from the horizontal midplane toward the horizontal endwalls, where they will be dissipated. A detailed analysis of the flow structure arising from a single RMF pulse is reported by Nikrityuk et al. ${ }^{[18]}$

In the present article, we consider the special case only if the RMF was switched off before completing the spinup process immediately at the end of the initial adjustment phase. Figures 3(a) and (b) show a transient flow pattern obtained by numerical simulations and UDV measurements in an experimental run, respectively, using an RMF pulse with the amplitude $B_{0}$ of $2.7 \mathrm{mT}$ and the pulse length $t_{p}$ of 3.6 seconds, which corresponds to the duration of the initial adjustment phase $t_{i a}$, for the case considered here. The colored contour plots display a sequence of the axial profiles of the vertical velocity. The amplitude of the velocity is represented by the color map. Drawing the particular velocity profiles along the ordinate and using the abscissa as a timeline, a spatiotemporal structure of the flow can be visualized. The comparison between the

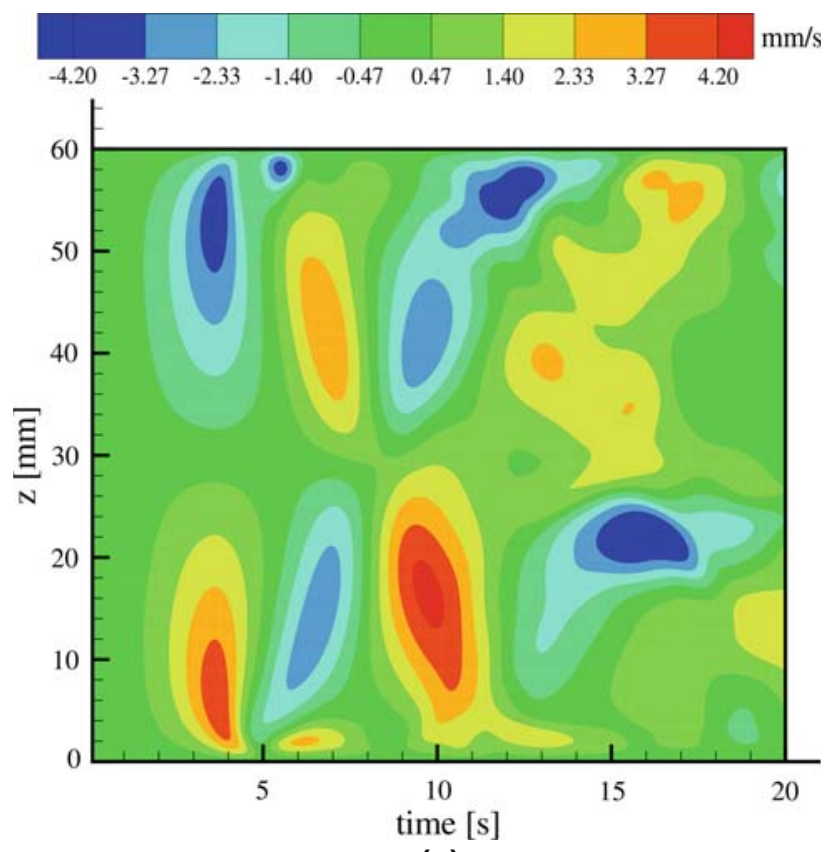

(a)

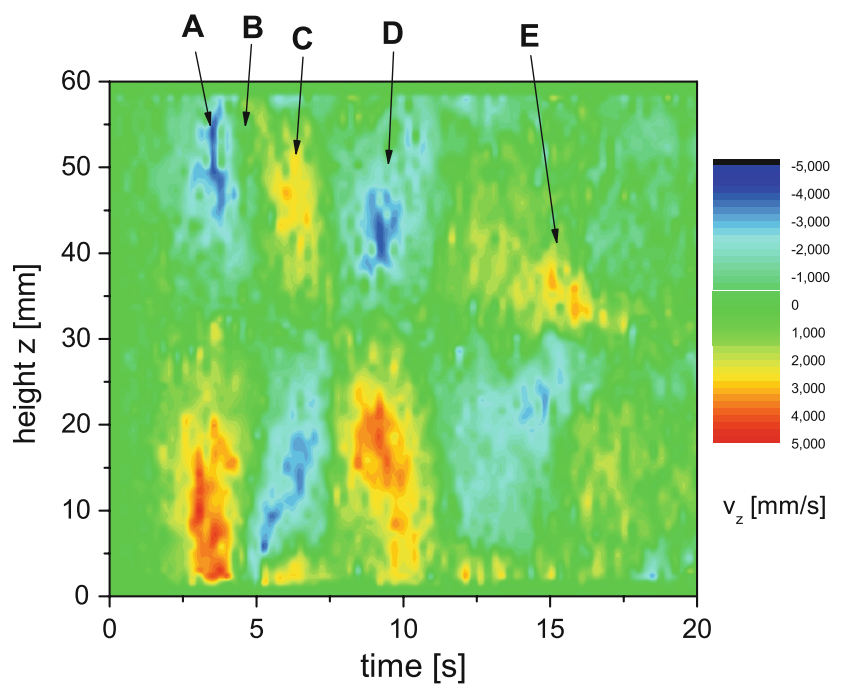

(b)

Fig. 3-Spatiotemporal plots of the vertical velocity showing a flow pattern resulting from an RMF pulse with $B_{0}=2.7 \mathrm{mT}$ : $(a)$ numerical simulation and $(b)$ velocity measurements recorded at $r / R_{0}=0.6$, where A through $\mathrm{E}$ are selected time marks for showing specific flow structures.

numerical and experimental results shows an excellent agreement. At the beginning, a descending flow appears in the upper part of the container and an ascending motion appears in the lower part, which indicates the formation of two toroidal vortices usually appearing during the initial adjustment phase. The shutdown of the RMF at $t_{p}=3.6 \mathrm{~s}$ (mark A) does not extinguish the secondary flow immediately. The double vortex structure undergoes oscillations; specifically, the double vortex decays temporarily (mark B) and will be displaced by another vortex pair emerging at the endwalls and spreading toward the midplane of the container 


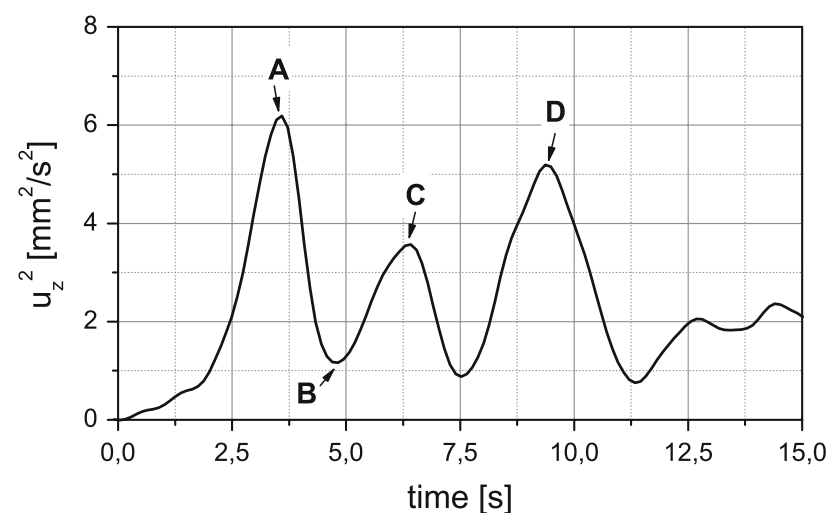

(a)

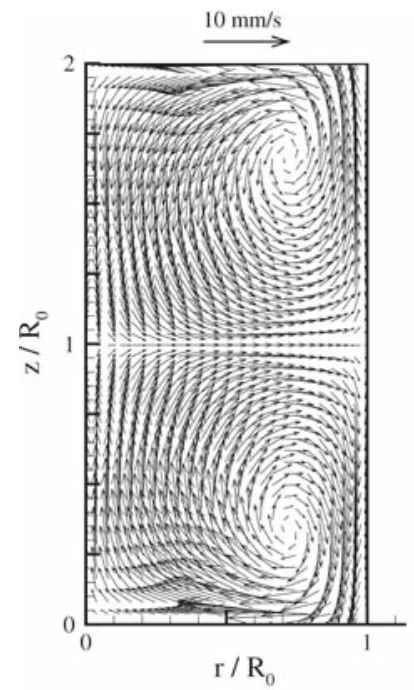

(b)

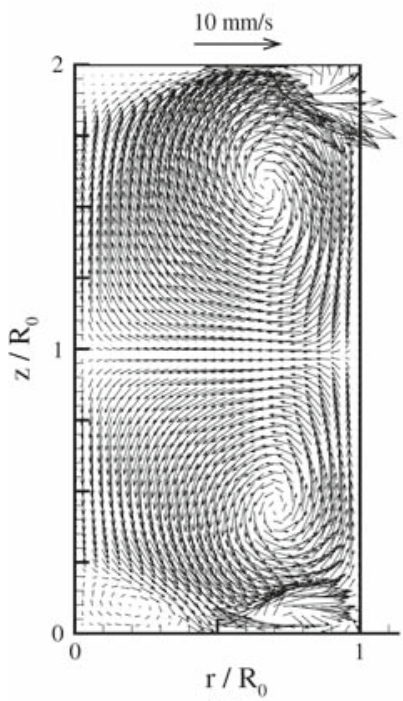

(d)

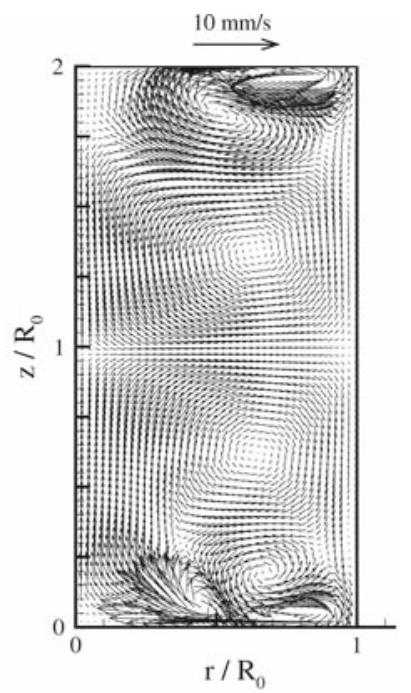

(c)

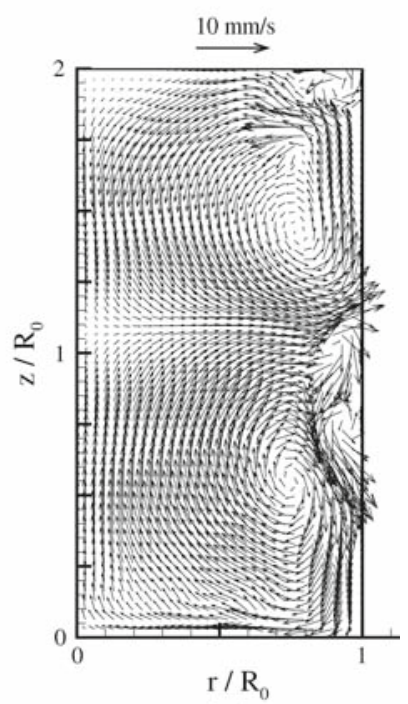

(e)
Fig. 4-Flow pattern resulting from an RMF pulse with $B_{0}=2.7 \mathrm{mT}$ : (a) $U_{z}^{2}$ averaged over the velocity profile (experimental result) and vector plots of the meridional velocity (numerical results) for the times (b) $3.6 \mathrm{~s}$ (point A), (c) $5 \mathrm{~s}$ (point B), (d) $6.3 \mathrm{~s} \mathrm{(point} \mathrm{C),} \mathrm{and} \mathrm{(e)} 9.5 \mathrm{~s} \mathrm{(point} \mathrm{D).}$

(mark C). It is important to note that the velocity shows a reversal of its direction. At the midplane, a short-time erasement of the flow structure seems to occur before
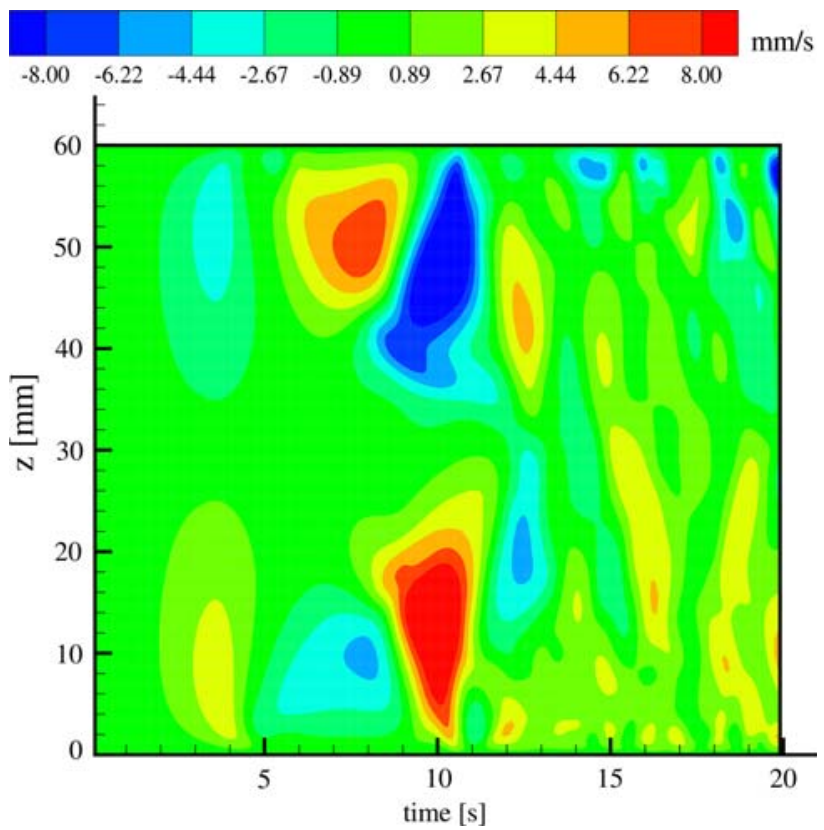

(a)

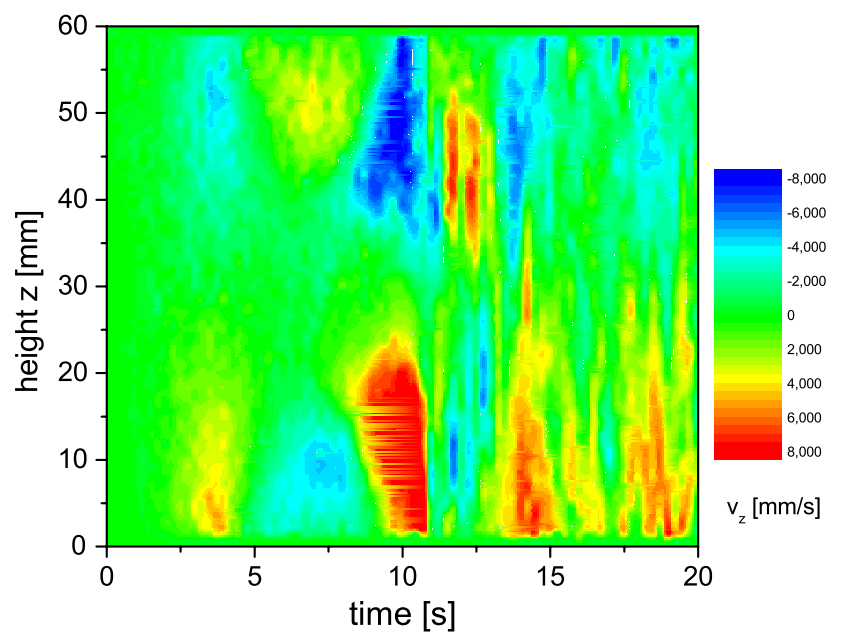

(b)

Fig. 5-Spatiotemporal plots of the vertical velocity showing a flow pattern resulting from an RMF pulse with $B_{0}=2.7 \mathrm{mT}$ followed by an inversion of the direction of rotation: $(a)$ numerical simulation and $(b)$ velocity measurements recorded at $r / R_{0}=0.6$.

the vortices expand over the other half of the container (mark D). Valentine and Miller ${ }^{[24]}$ studied the spindown of the flow inside a rotating cylinder numerically. Our results provide a good coincidence with their calculations, which predict the propagation of a so-called "solitary" wave from each endwall toward the midplane of the cylinder. Whereas the velocities in this period reach the same magnitude as those observed at the end of the initial adjustment phase, additional vertical structures appear with decreasing intensity (mark E).

Figure 4(a) contains a diagram showing the square value of the vertical velocity $U_{z}$ averaged along the measuring line, giving an indication concerning the intensity of the meridional circulation: 


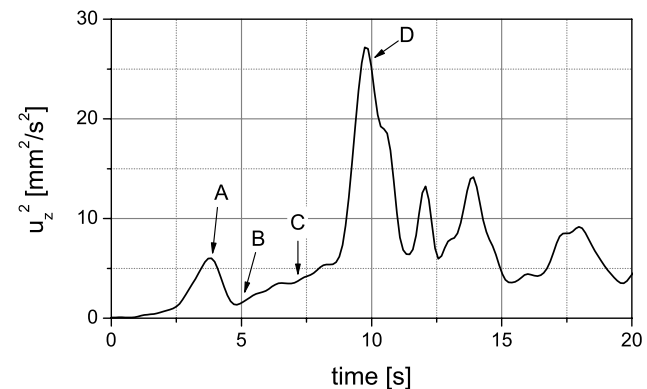

(a)

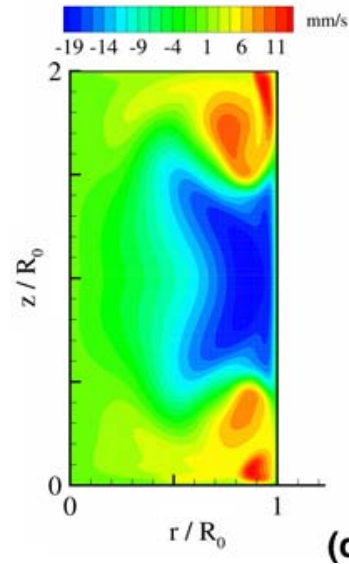

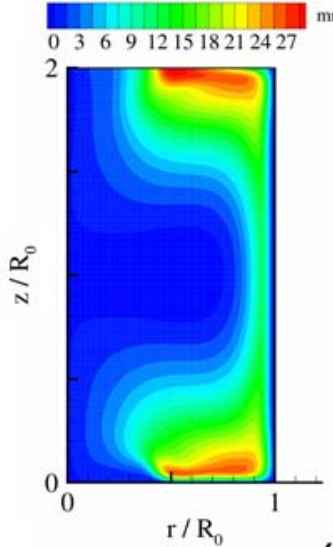

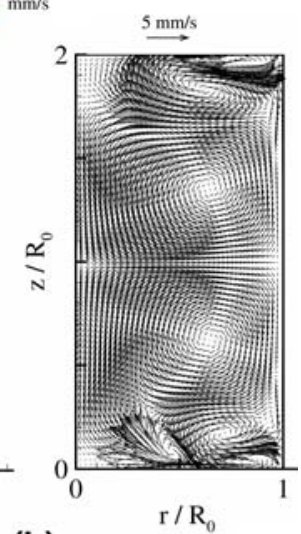

(b)

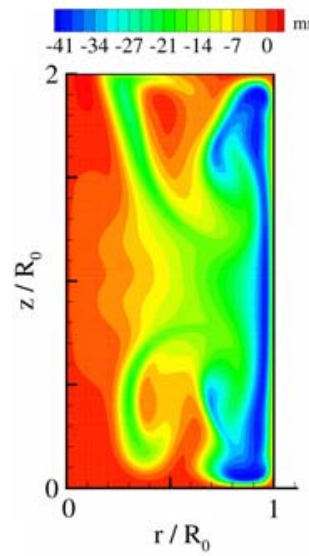

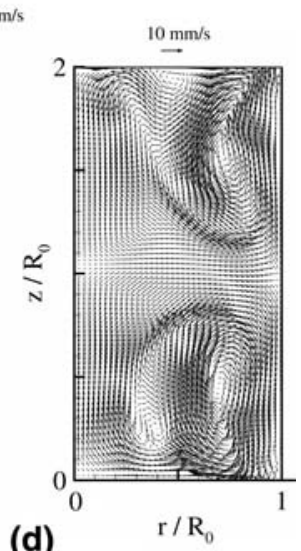

Fig. 6-Flow pattern resulting from an RMF pulse with $B_{0}=2.7 \mathrm{mT}$ followed by an inversion of the direction of rotation: $(a) U_{z}^{2}$ averaged over the velocity profile (experimental result), contour plots of the azimuthal velocity (numerical results), and vector plots of the meridional velocity (numerical results) for the times (b) $5 \mathrm{~s}$ (point B), (c) $7.2 \mathrm{~s}$ (point C), and (d) $10 \mathrm{~s}$ (point D).

$$
U_{z}^{2}=\frac{1}{H_{0}} \int_{0}^{H_{0}} u_{z}^{2} d z
$$

Corresponding snapshots of the meridional flow obtained from the numerical simulations are shown in Figures 4(b) through (e) for the time marks A, B, C, and $\mathrm{D}$, respectively. The maxima of the intensity of the meridional flow (marks A, C, and D) coincide with the appearance of the large vortical structures. The initial adjustment time $t_{\text {i.a. }}$ denotes the time period until the first maximum of $U_{z}^{2}$ in Figure 4(a) occurs, i.e., $t_{\text {i.a. }}=$ $t(A)$.

Following the idea of applying a pulse-shaped RMF, we have performed a similar experiment in which the magnetic field was not turned off but the sense of rotation of the field vector was inverted after the first RMF pulse. The modulation scheme is shown in Figure 2(b). Experimental and numerical results revealing the resulting flow pattern can be found in Figures 5 and 6 . There is a continuous input of energy into the system, but if the direction of the driving force was clockwise during the initial pulse, it changed to coun- terclockwise afterward. The reorientation of the flow direction does not occur simultaneously at different heights of the cylinder, resulting temporarily in strong vertical gradients of the azimuthal velocity. This effect becomes obvious from the numerical simulations of the azimuthal flow presented in the Figures 6(b) through (d). As already discussed, with respect to the spinup process, such gradients in the angular motion drive the secondary, recirculating flow. Consequently, after the inversion of the driving force, the vertical velocity clearly exceeds the values occurring in the initial double vortex (Figures 5 and 6(a)). These strong oscillations decay quickly and the flow undergoes the spinup process described at the beginning of this section, until the secondary flow reaches a quasi-steady state.

\section{THE RMF-PSCD}

Section V showed the occurrence of strong velocity fluctuations and an amplification of the secondary flow arising from an impulsive change in the amplitude or the direction of the electromagnetic force, respectively. Moreover, periodic reversals of the direction of the secondary flow have been observed. It can be assumed 


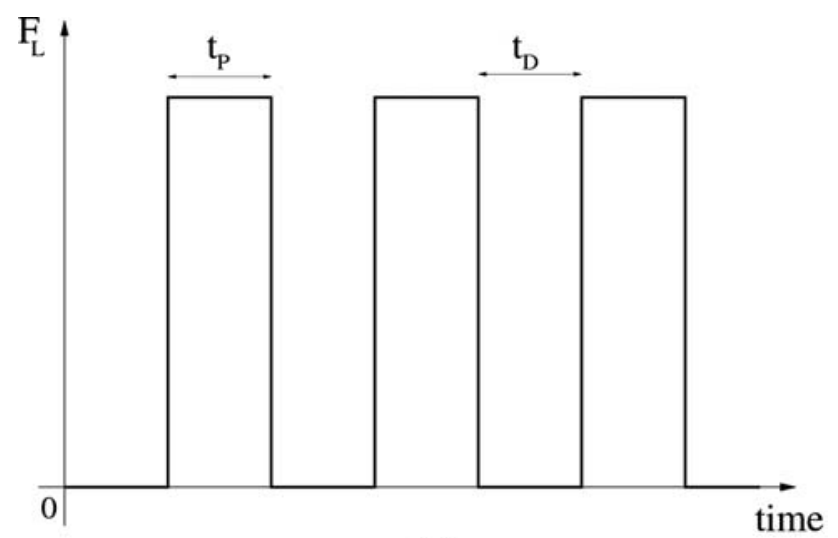

(a)

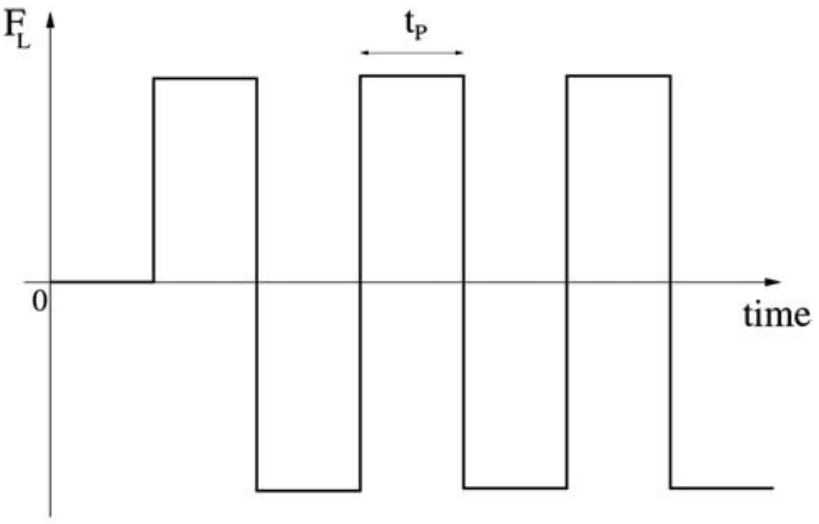

(b)

Fig. 7-Temporal scheme for the modulation of the electromagnetic force: (a) RMF-PSCD regime (b) RMF-PSAD regime.

that such features of the velocity field could be beneficial for enforcing the mixing of the melt or for mitigating the segregation during solidification. To be attractive for technological applications, this strong, oscillating behavior - especially of the secondary, recirculating flow - has to be maintained over a longer time periodfor example, for a few minutes. This behavior may lend itself to meeting that goal by a repeated switching on and off of the RMF, i.e., the generation of a succession of RMF pulses, as shown in Figure 7(a). In the same way, the rotating direction of the RMF can be inverted, as shown in Figure 7(b) and discussed in Section VII. Additional control variables appear, namely, the pulse length $t_{p}$ and the time between the pulses $t_{d}$, also called the dormant period.

In the present study, exclusively equal periods of driving and dormant phases are used. Thus, we consider the dependence of the intensity of the secondary flow merely on the magnetic-field strength $B_{0}$ and the pulse frequency $f_{p}=1 /\left(2 \cdot t_{p}\right)$.

Figure 8 displays spatiotemporal plots of the vertical velocity obtained for RMF pulses with a magnetic-field strength of $5.8 \mathrm{mT}$. The variation of the pulse frequency $f_{p}$ shows a distinct influence on the secondary flow. In the case of low pulse frequencies (for example, $f_{p}=0.2$ $\mathrm{Hz}$ in Figure 8(a)), the flow pattern is characterized by a noncontinuous double vortex structure appearing with

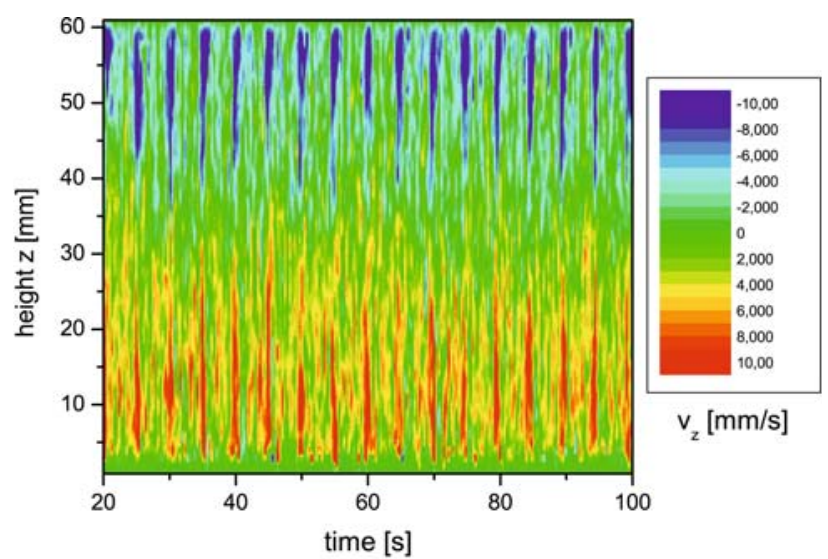

(a)

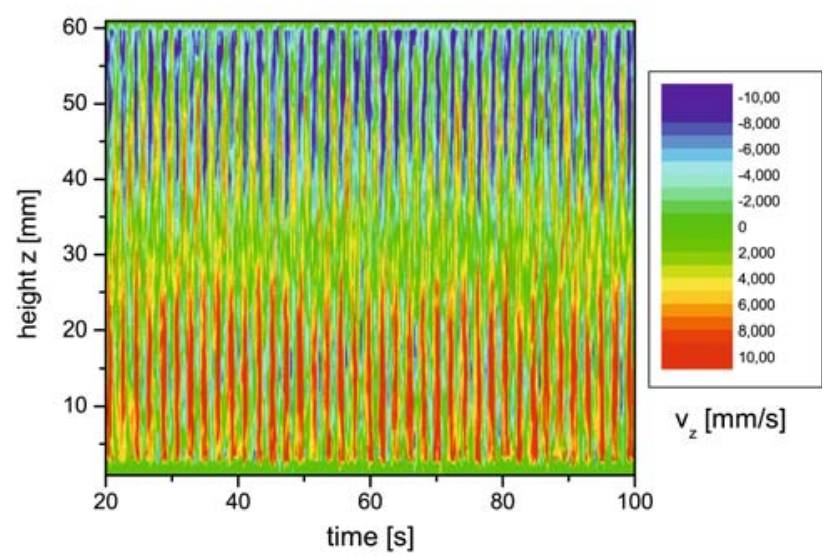

(b)

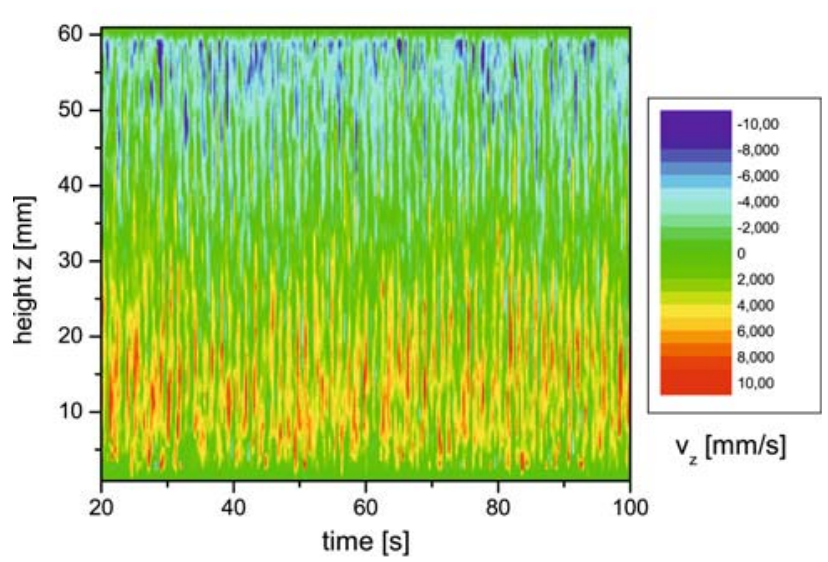

(c)

Fig. 8- Spatiotemporal plots of the vertical velocity for RMF-PSCD regime, $B_{0}=5.8 \mathrm{mT}$ : (a) $f_{p}=0.2 \mathrm{~Hz},(b) f_{p}=0.475 \mathrm{~Hz}$, and $(c)$ $f_{p}=0.77 \mathrm{~Hz}$.

a periodicity $f_{p}$. The interim time is characterized by velocity oscillations at lower intensities. Reversals of the flow direction can be observed sporadically, but the original direction of the secondary flow obviously prevails. In the experiment, the pulse frequency was incremented in a stepwise manner. A new type of flow pattern has been found for a pulse frequency of $0.475 \mathrm{~Hz}$ (Figure 8(b)). A pronounced double-vortex structure appears that shows a periodic inversion of the 


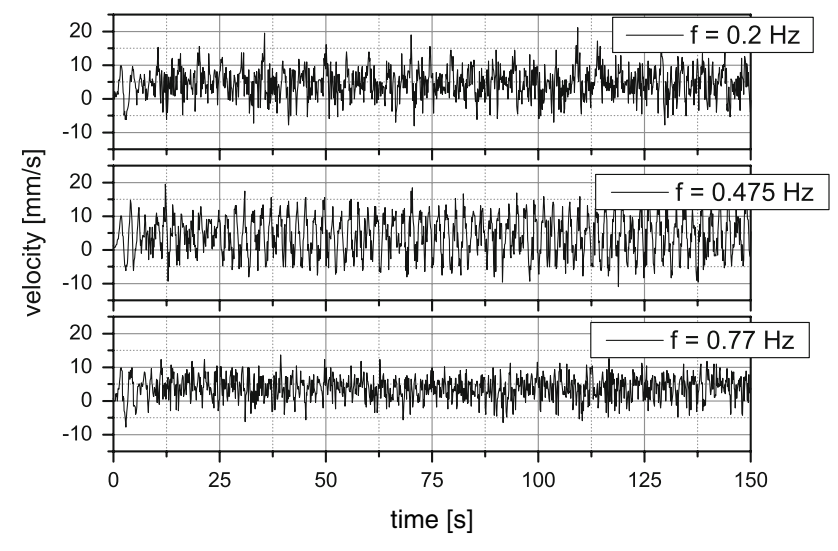

Fig. 9-Time series of the vertical velocity at $z=10 \mathrm{~mm}$ and $r$ / $R_{0}=0.6$ (RMF-PSCD regime, $B_{0}=5.8 \mathrm{mT}$ ).

sign of vorticity. Note that such a flow pattern can only be observed for a very narrow range of frequencies around the value of $0.475 \mathrm{~Hz}$, for the particular field strength of $5.8 \mathrm{mT}$. An additional increase of $f_{p}$ implies a weakening of the secondary flow. The situation can also be illustrated by the time series of the local velocity contained in Figure 9. The time series were recorded at a vertical position of $10 \mathrm{~mm}$ above the bottom of the fluid container, for the same radial position and RMF parameters as shown in Figure 8. Adjusting the frequency of $0.475 \mathrm{~Hz}$ provides regular oscillations of the vertical velocity. Moreover, the amplitude of the velocity oscillations exceeds the values obtained for both smaller and higher pulse frequencies. Thus, an optimal value for the pulse frequency has been identified. An explanation of this phenomenon requires an understanding of the interaction between the primary and the secondary flow. The energy transfer between the rotary motion and the meridional flow is governed by inertial waves. Recently, the importance of these waves was addressed in the context of RMF-driven fluids. ${ }^{[17,25]}$ Preliminary results obtained by a detailed analysis of the fluid mechanical aspects reveal that a characteristic flow pattern, as shown in Figure 8(b), will be achieved if the pulse frequency $f_{p}$ corresponds to the eigenperiod of inertial waves in a developed regime, as given by Greenspan. ${ }^{[26]}$

Additional measurements and numerical calculations have been performed to investigate the dependence of the optimal value of the pulse frequency on the magnetic-field induction $B_{0}$. It has been found that the optimum of $f_{p \text {,optimum }}=1 /\left(2 \cdot t_{p \text {,optimum }}\right)$ correlates with an optimum pulse length of $t_{p \text {,optimum }}=t(B)-t(A)$, which means that the duration of the individual pulses has to coincide with the time period occurring between the first minimum and maximum of $U_{z}^{2}$, as shown in Figure 4(a).

\section{THE RMF-PSAD}

Another method of stirring can be realized by applying an alternately-pulsed RMF, i.e., the sense of rotation will be periodically inverted. The alternating course of the Lorentz force $F_{L}$, which drives the fluid motion, is shown in Figure 7(b). We have already seen in Section $\mathrm{V}$ that distinctive oscillations of the secondary flow can be expected here with higher velocity amplitudes, as observed for the RMF-PSCD. It is self-evident that the pulse frequency $f_{p}=1 /\left(2 \cdot t_{p}\right)$ should have considerable impact on the resulting flow structure for the RMF-PSAD regime, as well.

The respective spatiotemporal plots of the vertical velocity recorded for different frequencies $f_{p}$ at a magnetic-field strength of $2.7 \mathrm{mT}$ are depicted in Figure 10. Figure 10(a) shows a typical situation when relatively long cycles $\left(t_{p}=25 \mathrm{~s}\right.$, in the case under consideration) have been selected. High intensities of the secondary flow emerge directly after changing the direction of the RMF. However, these large velocity values are efficiently damped, because of the stabilizing effect arising from the upcoming rotary motion of the liquid metal. Subsequently, the fluid experiences the usual spinup process until the next reversal of the rotational direction will be initiated. The interval between the reversals was reduced until a frequency of $0.08 \mathrm{~Hz}$ was reached. The corresponding velocity field in Figure 10(b) reveals strong fluctuations and permanent changes of the sign of the velocity. The flow appears rather complex; a stationary vortex structure cannot be identified. A considerable decrease in the flow intensity becomes apparent for frequencies above $0.1 \mathrm{~Hz}$ (as an example, Figure $10(\mathrm{c})$, for $f_{p}=0.2 \mathrm{~Hz}$ ). Due to its own inertia, the fluid can no longer follow the alternating forcing. To get a measure of the intensity of the secondary flow, the square value of the vertical velocity $u_{z}^{2}$ was averaged over both the axial line where the velocity profiles have been determined and the total measuring time $T$ :

$$
\bar{U}_{z}^{2}=\frac{1}{T} \int_{0}^{T} \frac{1}{H_{0}} \int_{0}^{H_{0}} u_{z}^{2} d z d t
$$

Figure 11 shows a diagram in which the resulting mean values $\bar{U}_{z}^{2}$ are drawn $v s$ the frequency $f_{p}$. Similar to the findings for the RMF-PSCD regime, it becomes obvious that pronounced maxima of $\bar{U}_{z}^{2}$ occur at selective frequencies, varying for different values of the magnetic-field strength $B_{0}$. Note that the location of the maxima corresponds to an optimal pulse length $t_{p}=$ $t(A)=t_{i . a}$. Whereas the intensity of the secondary flow at lower frequencies shows a level comparable to a permanently-applied RMF, a drastic decay of the secondary flow has to be noticed at higher frequencies.

In the present study, it was not planned to measure the form of the free surface quantitatively during the stirring. However, the experimental configuration allowed for a visual observation in parallel with the velocity measurements. In a simple, supplementary experiment, a continuously-applied RMF was compared with the stirring using the RMF-PSAD regime. A magnetic-field strength of $8.3 \mathrm{mT}$ was chosen for the continuous RMF. Here, a distinct deflection of the free surface could be observed easily. Then, the melt was 


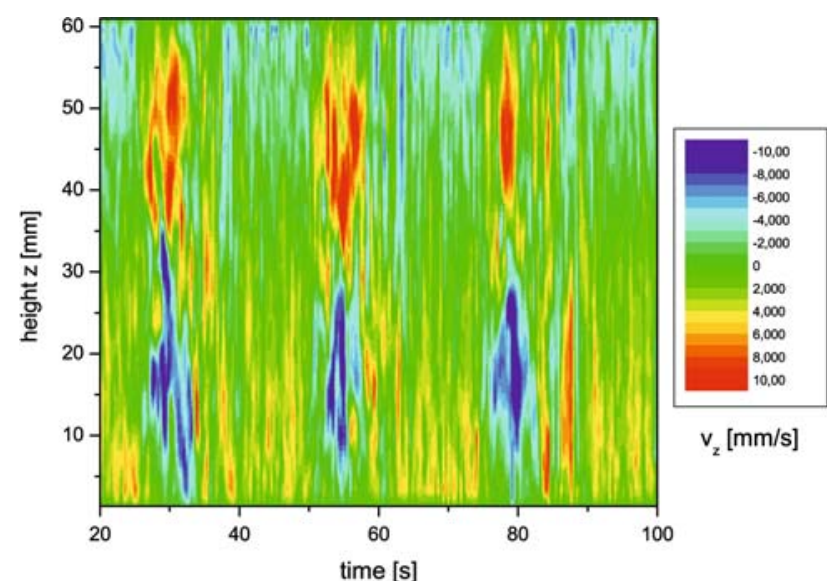

(a)

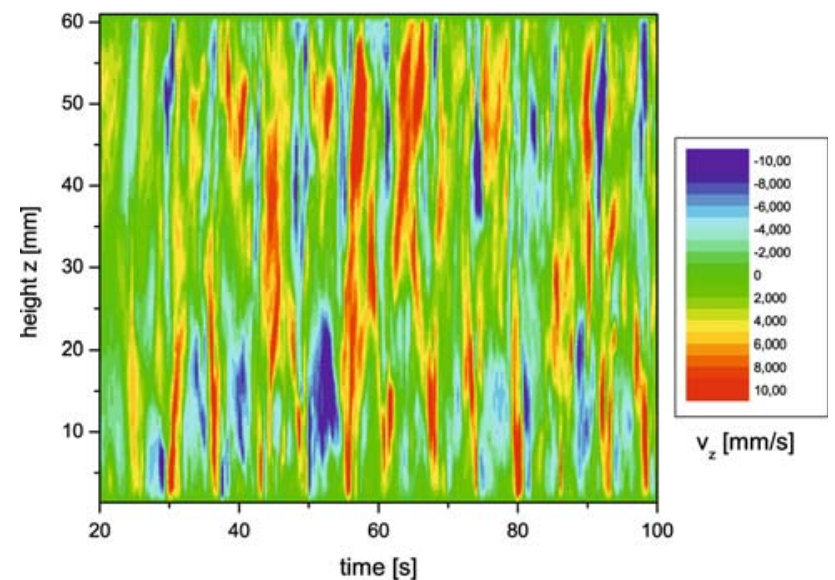

(b)

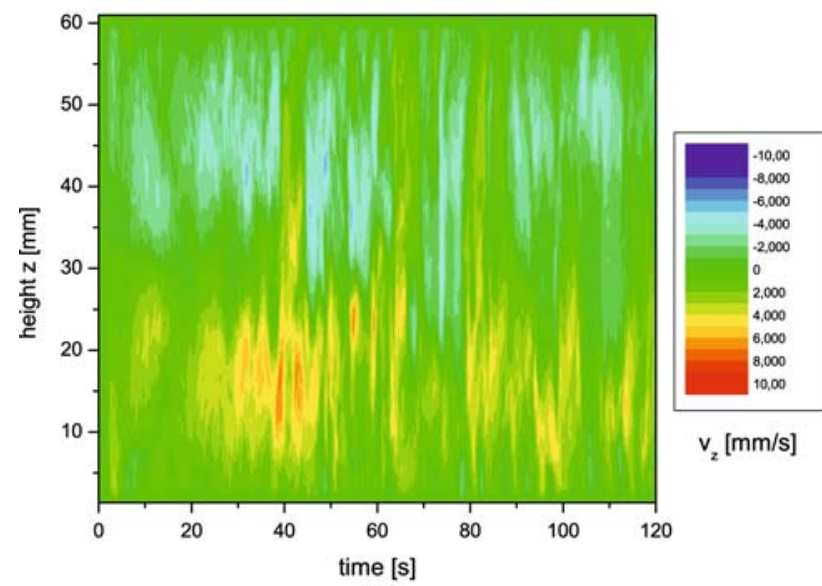

(c)

Fig. 10 - Spatiotemporal plots of the vertical velocity: RMF-PSAD regime, $B_{0}=2.7 \mathrm{mT}$ : (a) $f_{p}=0.02 \mathrm{~Hz},(b) f_{p}=0.08 \mathrm{~Hz}$, and $(c)$ $f_{p}=0.2 \mathrm{~Hz}$

stirred by means of an alternately-pulsed magnetic field with $B_{0}$ of $4.4 \mathrm{mT}$ and a frequency $f_{p}$ of $0.15 \mathrm{~Hz}$. For that case, the velocity measurements showed nearly the same intensity of the vertical velocity, in terms of the value $\bar{U}_{z}^{2}$. The respective spatiotemporal plots of the vertical velocity can be found in Figure 12. Whereas

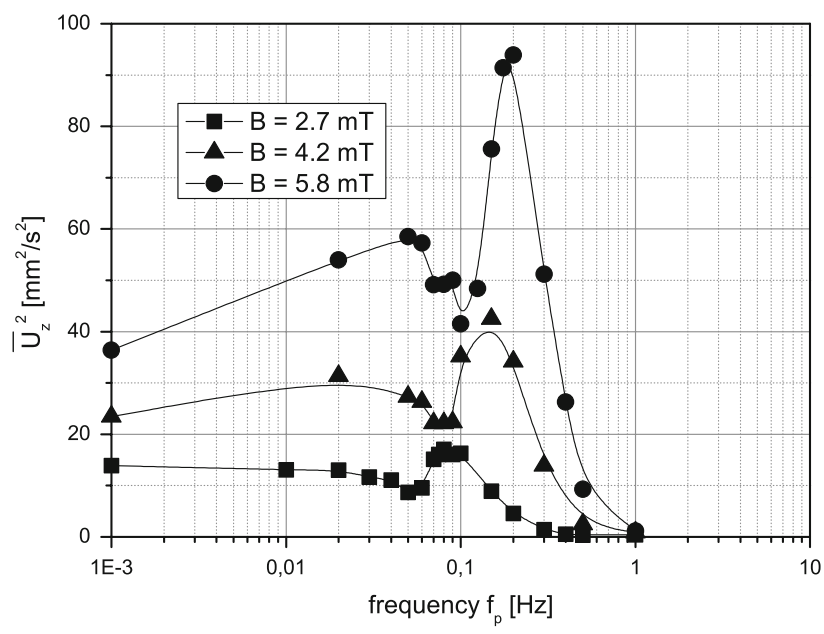

Fig. 11-Dependence of the intensity of the secondary flow on the frequency of reversals $f_{p}$ of rotational direction: $\bar{U}_{z}^{2}$ is the average value over the velocity profile and the measuring time.

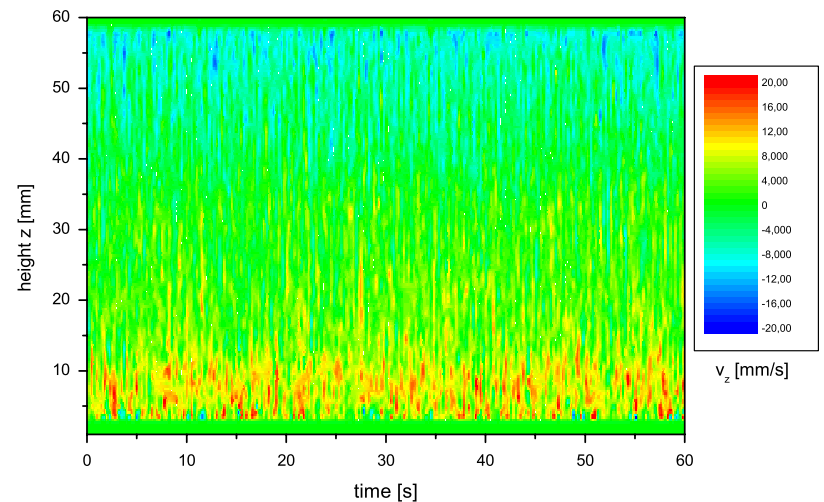

(a)

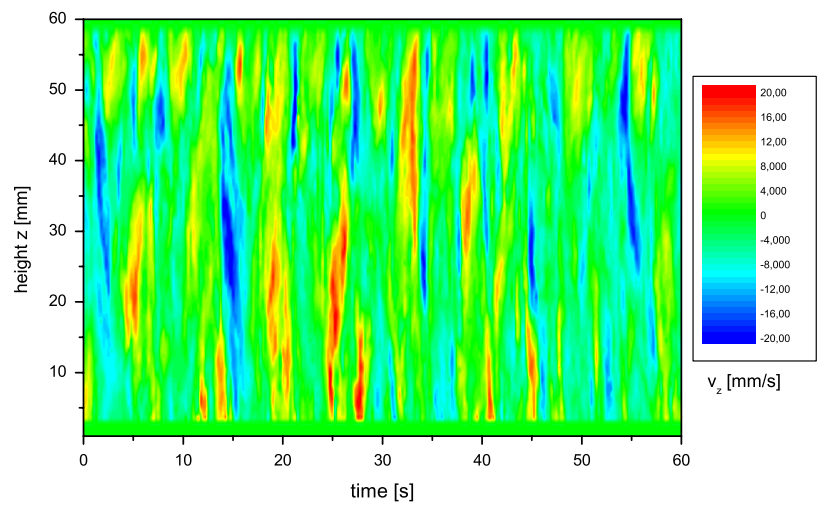

(b)

Fig. 12 - Spatiotemporal plots of the vertical velocity: (a) continuously-applied RMF, and $B_{0}=8.3 \mathrm{mT}$; and $(b)$ RMF-PSAD regime, $B_{0}=4.4 \mathrm{mT}$, and $f_{p}=0.15 \mathrm{~Hz}$.

the stirring with the continuous RMF causes distinct perturbations of the free surface, an almost planar shape of the surface can be maintained in the case of the alternately-pulsed field (Figure 13). 


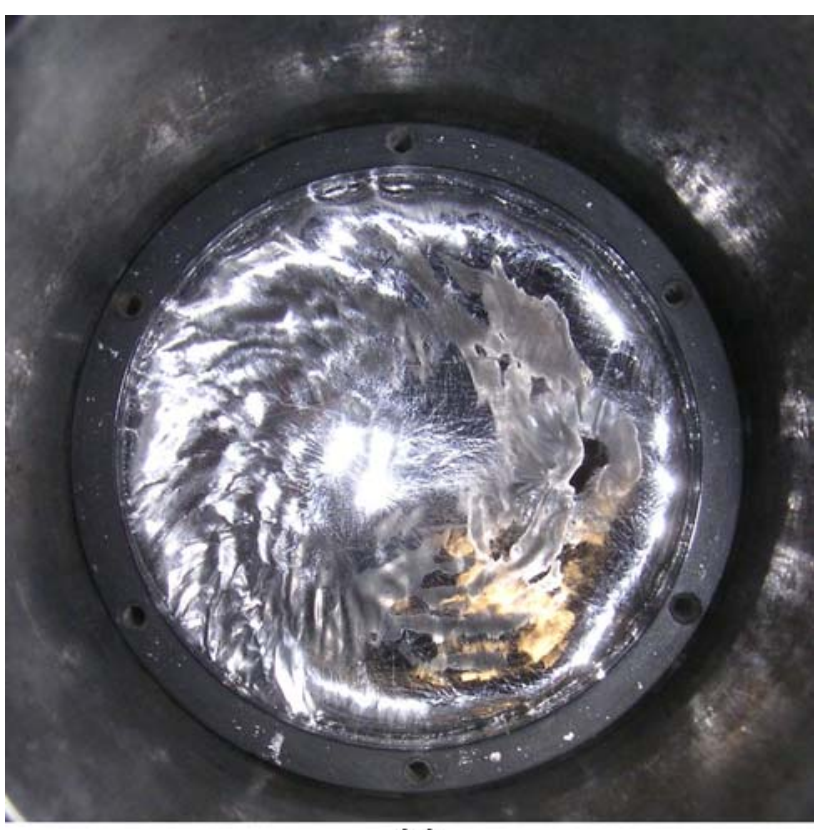

(a)

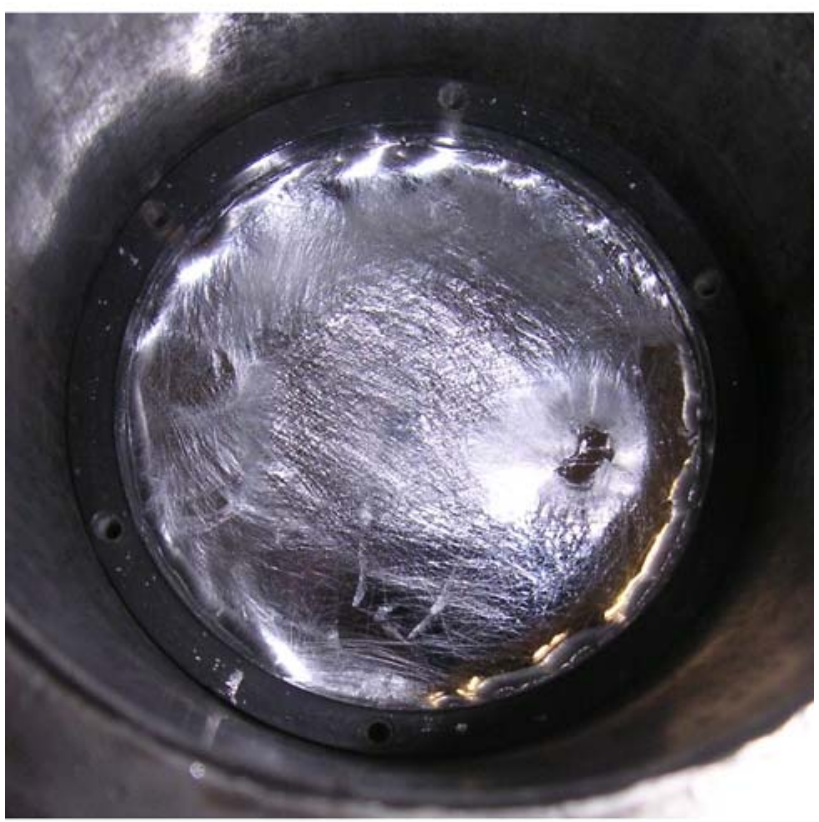

(b)

Fig. 13-Photographs of the free surface of the melt during stirring: (a) continuously-applied RMF, and $B_{0}=8.3 \mathrm{mT}$; and (b) RMFPSAD regime, $B_{0}=4.4 \mathrm{mT}$, and $f_{p}=0.15 \mathrm{~Hz}$.

\section{DISCUSSION}

Our approach to achieving an efficient mixing in the melt is based on a considerable amplification of the meridional, secondary flow, without additional acceleration of the primary, rotary motion. In comparison with a continuously-applied RMF, the same or even a higher mixing effect can be realized, accompanied by lower energy consumption. The flow measurements show the capability of overcoming the limited mixing character of conventional rotary stirring, specifically, of providing a vigorous mixing in the bulk of a liquid metal column, only in connection with a considerable deformation of the upper surface. Both stirring regimes studied in the present article generate high mixing intensities through a distinct intensification of the secondary flow, which reduces the mixing time of the process under consideration, but leaves the free surface almost unaffected. These features make the electromagnetic stirring by means of RMF pulses very attractive for various industrial applications in which RMFs are already applied for melt agitation. For example, specific stirring technologies have been proposed for steelmaking processes using two coil systems. ${ }^{[4,14]}$ The method presented here requires only one coil system and consumes a small amount of electric energy. Furthermore, the secondary flow can be organized in such a way that periodic reversals of the flow direction occur. This feature becomes important with respect to an application for unidirectional solidification of metal alloys, because it offers the potential for preventing macrosegregation.

The outcome of the present study demonstrates that a preferential intensification of the secondary flow requires the careful adjustment of the respective frequencies $f_{p}$ for the pulsing and the inversion of the rotational direction of the RMF. The respective optimum for $f_{p}$ depends on the magnetic-field strength $B_{0}$, the material properties of the liquid, and the geometry under consideration. A mismatch of the relevant parameters may prevent an improvement of the mixing quality; actually, the results may become worse compared to the case of a continuously-applied RMF. An important finding is that the optimum conditions for the stirring can be achieved if the pulse length $t_{p}$ is chosen to be $t(B)$ - $t(A)$ and $t(A)$ for the RMF-PSCD and the RMFPSAD regime, respectively. It is worth noting that the characteristic time scales $t(A)=t_{i a}$ and $t(B)$, known from the fluid mechanical studies of the RMF spinup ${ }^{[17]}$ or the single RMF pulse, ${ }^{[18]}$ play a significant role in the stirring regimes studied here. This finding can be used as a kind of recipe for discovering the ideal stirring frequencies $f_{p}$; however, the characteristic time scales have to be specified separately, with respect to the particular experimental configuration.

\section{CONCLUSIONS}

This article presents a tailored method of electromagnetic stirring. An RMF acts upon a column of liquid metal and the amplitude of the field strength has been modulated in two different ways: a sequence of RMFPSCD pulses (Section V) and a pulse sequence of RMFPSAD, in which the direction of rotation of the field is inverted periodically (Section VI). Numerical calculations and model experiments showed very good agreement with respect to the resulting flow structures.

The electromagnetic stirring method that uses a timemodulated RMF offers considerable potential for a wellaimed modification of casting properties, also. Both the RMF-PSCD and the RMF-PSAD stirring regimes enable us to avoid macrosegregations, by inducing periodic reversals of the recirculating, secondary flow. 
Additional research activities on this subject are under progress. The application during the directional solidification of Al-Si alloys is the subject of Part II of this article.

\section{ACKNOWLEDGMENTS}

This work was financially supported by Deutsche Forschungsgemeinschaft (DFG) in the form of the collaborative research center SFB 609, "Electromagnetic Flow Control in Metallurgy, Crystal Growth and Electrochemistry.'"

\section{NOMENCLATURE}

$B_{0} \quad$ magnetic induction

$J \quad$ Bessel function

$U_{z}^{2} \quad$ square value of the vertical velocity averaging along the $z$ axis

$\bar{U}_{z}^{2} \quad$ time-averaged value of $U_{z}^{2}$

$f_{p} \quad$ pulse frequency

$t_{p} \quad$ pulse length

$t_{i a} \quad$ duration of the initial adjustment phase

$F_{L} \quad$ Lorentz force

$H_{0} \quad$ height of cylinder

$R_{0} \quad$ radius of cylinder

$r, \theta, z \quad$ cylindrical coordinate axes

u velocity

$S \quad$ shielding parameter

$p \quad$ pressure

$N \quad$ magnetic interaction parameter

$\mathrm{Re}_{m}$ magnetic Reynolds number

\section{GREEK SYMBOLS}

$\omega$ angular frequency of the magnetic field

$\sigma \quad$ electrical conductivity

$\rho$ density

$\lambda \quad$ root of $J^{\prime}(x)=0$

$\mu_{0} \quad$ magnetic permeability

$v \quad$ kinematic viscosity

\section{REFERENCES}

1. P.A. Davidson: An Introduction to Magnetohydrodynamics, Cambridge University Press, Cambridge, United Kingdom, 2001, pp. 285-300.

2. K.-H. Spitzer, G. Reiter, and K. Schwerdtfeger: ISIJ Int., 1996, vol. 36, pp. 487-92.

3. K.-H. Spitzer: Prog. Cryst. Growth Charact. Mater., 1999, vol. 38, pp. 59-71.

4. F.-C. Chang, J.R. Hull, and L. Beitelman: Metall. Mater. Trans. $B$, 2004, vol. . 35B, pp. 1129-37.

5. D. Perrier, Y. Fautrelle, and J. Etay: Metall. Mater. Trans. B, 2003, vol. 34B, pp. 669-78.

6. C. Vives: Metall. Trans. B, 1993, vol. 24B, pp. 493-510.

7. J.K. Roplekar and J.A. Dantzig: Int. J. Cast Met. Res., 2001, vol. 14, pp. 79-95.

8. B. Willers, S. Eckert, U. Michel, I. Haase, and G. Zouhar: Mater. Sci. Eng. A, 2005, vol. 402, pp. 55-65.

9. J. Priede and G. Gerbeth: J. Cryst. Growth, 2005, vol. 285, pp. 261-69.

10. P.A. Nikrityuk, K. Eckert, and R. Grundmann: Int. J. Heat Mass Transfer, 2006, vol. 49, pp. 1501-15.

11. B. Willers, S. Eckert, P.A. Nikrityuk, and K. Eckert: in Modeling of Casting, Welding, and Advanced Solidification Processes-XI, C.A. Gandin and M. Bellot, eds., TMS, Warrendale, PA, 2006, pp. 333-40.

12. S. Steinbach and L. Ratke: Mater. Sci. Eng. A, 2005, vols. 413414, pp. 200-04.

13. P.A. Nikrityuk, K. Eckert, and R. Grundmann: Proc. Conf. Turbulence and Interactions TI2006, ONERA, Toulouse, 2006. CD.

14. S. Taniguchi, K. Mataike, M. Okubo, T. Ando, and K. Ueno: Proc. 4th Int. Conf. on Electromagnetic Processing of Materials (EPM2003), ISIJ, Tokyo, 2003, pp. 339-43.

15. S. Kojima, T. Ohnishi, T. Mori, K. Shiwaku, I. Wakasugi, and M. Ohgarni: 66th Steelmaking Conf., Atlanta, GA, ISS-AIME, Warrendale, 1983, pp. 127-31.

16. M. Ungarish: J. Fluid Mech., 1997, vol. 347, pp. 105-18.

17. P.A. Nikrityuk, M. Ungarish, K. Eckert, and R. Grundmann: Phys. Fluids, 2005, vol. 17, pp. 067101-1-067101-17.

18. P.A. Nikrityuk, S. Eckert, and K. Eckert: Eur. J. Mech. B/Fluids, 2007, doi: 10.1016/j.euromechflu.2007.05.004.

19. Y. Takeda: Nucl. Eng. Des., 1991, vol. 126, pp. 277-84.

20. D. Brito, H.-C. Nataf, P. Cardin, J. Aubert, and J.P. Masson: Exp. Fluids, 2001, vol. 31, pp. 653-63.

21. A. Cramer, C. Zhang, and S. Eckert: Flow Meas. Instrum., 2004, vol. 15 , pp. $145-53$.

22. L.M. Witkowski, J.S. Walker, and P. Marty: Phys. Fluids, 1999, vol. 11, pp. 1821-26.

23. J.H. Ferziger and M. Peric: Computational Methods for Fluid Dynamics, Springer, New York, NY, 2002, pp. 196-200.

24. D.T. Valentine and K.D. Miller: Phys. Fluids, 1994, vol. 6, pp. 1535-47.

25. P.A. Davidson and F. Boysan: Ironmaking and Steelmaking, 1991, vol. 18 , pp. $245-52$.

26. H.P. Greenspan: The Theory of Rotating Fluids, Breukelen Press, Brooklyn, NY, 1990, pp. 81-85. 